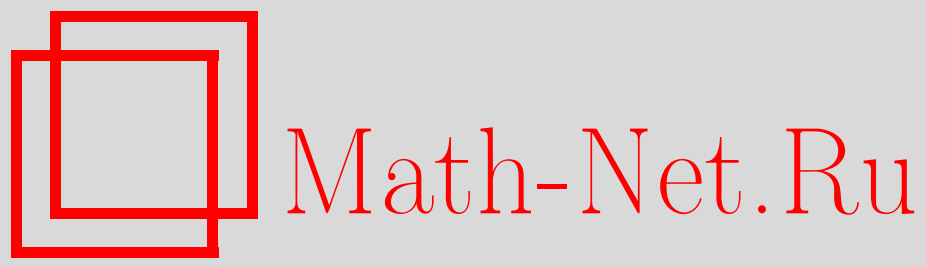

В. И. Хохлов, Многочлены, ортогональные относительно полиномиального распределения, и факториальностепенной формализм, Теория вероятн. и ее примен., 2001, том 46, выпуск 3, 585-592

DOI: https://doi.org/10.4213/tvp3907

Использование Общероссийского математического портала Math-Net.Ru подразумевает, что вы прочитали и согласны с пользовательским соглашением

http://www . mathnet.ru/rus/agreement

Параметры загрузки:

IP: 54.92 .164 .108

26 апреля 2023 г., 14:59:54

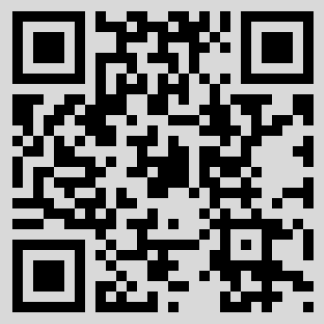




\title{
REFERENCES
}

1. Goll T., Kallsen J. Optimal portfolios for logarithmic utility. - Stochastic Process. Appl., 2000, v. 89, p. 31-48.

2. Jacod J. Calcul stochastique et problèmes de martingales. Berlin-Heidelberg: Springer-Verlag, 1979, 539 p.

3. Jacod $J$. Intégrales stochastiques par rapport à une semi-martingale vectorielle et changements de filtration. - Lecture Notes in Math., 1980, v. 784, p. 161-172.

4. Jacod J., Shiryaev A. Limit Theorems for Stochastic Processes. Berlin-Heidelberg: Springer-Verlag, 1987. Рус. перев.: Жаход Ж., Ширяев A. Н. Предельные теоремы для случайных процессов. Т. 1, 2. М.: Физматлит, 1994, 544 с; 368 с.

5. Kallenberg $O$. Some time change representations of stable integrals, via predictable transformations of local martingales. - Stochastic Process. Appl., 1992, v. 40, № 2, p. 199-223.

6. Revuz D., Yor M. Continuous Martingales and Brownian Motion. Berlin: SpringerVerlag, 1999, $602 \mathrm{p}$.

7. Rosiński J., Woyczyński $W$. On Itô stochastic integration with respect to $p$-stable motion: Inner clock, integrability of sample paths, double and multiple integrals. Ann. Probab., 1986, v. 14, p. 271-286.

8. Samorodnitsky G., Taqqu M. Stable Non-Gaussian Random Processes: Stochastic Models with Infinite Variance. New York: Chapman\&Hall, 1994, 632 p.

9. Sato K. Lévy Processes and Infinitely Divisible Distributions. Cambridge: Cambridge Univ. Press, 1999, 486 p.

10. Ширяев $A$. Н. Основы стохастической финансовой математики. Т. 1, 2. М.: Фазис, 1998, 512 с.; 544 с. Англ. перев.: Shiryaev A. Essentials of Stochastic Finance. Singapore: World Sci. Publ., 1999.

Поступила в редакцию

4.V.2000

(C) $2001 \mathrm{r}$.

ХоХлов в. и.*

\section{МНОГОЧЛЕНЫ, ОРТОГОНАЛЬНЫЕ ОТНОСИТЕЛЬНО ПОЛИНОМИАЛЬНОГО РАСПРЕДЕЛЕНИЯ, И ФАКТОРИАЛЬНО-СТЕПЕННОЙ ФОРМАЛИЗМ}

\begin{abstract}
Ключевые слова и фразы: биномиальное распределение, пуассоновское распределение, полиномиальное распределение, многочлены Кравчука, многочлены Пуассона-Шарлье, факториально-степенные многочлены, ортогональные относительно полиномиального распределения многочлены, комбинаторные тождества, тождество Лерха.
\end{abstract}

Введение. Первоначальный вариант этого сообщения был направлен в редакцию журнала «Теория вероятностей и ее применения» еще в начале 1985 года. Однако в том варианте отсутствовали указания на иллюстрации таких применений полученных результатов и предложенной комбинаторной техники, которые могли бы представлять чисто вероятностный интерес. Поэтому автор решил в конечном счете ограничиться публикацией формулировок главных результатов в тезисах [6]. Впоследствии к ним были даны комментарии в докладе на IV Всемирном конгрессе Обшества им. Бернулли в Вене (август 1996 г.), а в докладе на Международном

* Математический институт им. В. А. Стеклова РАН, ул. Губкина, 8, 117966 Москва, ГСП-1, Россия; e-mail: tvp@tvp.ru 
конгрессе математиков в Берлине (август 1998 г.) был приведен пример практического использования данной техники в области дискретной математики. Недавно обнаружилось, что разработанная техника оказалась достаточно удобной для получения пуассоновского [1] и биномиальных [2] аналогов неравенства Чернова [4]. Это позволяет теперь предложить вниманию читателя немного расширенный и переработанный вариант той старой заметки.

Автор считает своим приятным долгом поблагодарить Ю. В. Прохорова, Б. А. Севастьянова, Ю.В. Болотникова, О. В. Вискова за разносторонние обсуждения результатов и - за ряд сушественных поправок - рецензента.

Систему $\left\{P_{\alpha}(\mathbf{x}), \mathbf{x} \in \mathbf{R}^{l}\right\}_{\alpha=0,1, \ldots}$ многочленов в $\mathscr{L}_{2}(\boldsymbol{\mu})$, где $\boldsymbol{\mu}$ - заданная мера на $\mathbf{R}^{\prime}(l=1,2, \ldots)$, обладающую свойством

$$
\int P_{\alpha}(\mathbf{x}) P_{\beta}(\mathbf{x}) d \mu(\mathbf{x})=\delta_{\alpha \beta}, \quad \alpha, \beta=0,1, \ldots
$$

( $\delta_{\alpha \beta}$ - символ Кронекера), называют системой многочленов, ортогональных относительно распределения $d \mu(\mathrm{x})$.

Хорошо известно [8], что для частного случая полиномиального распределения биноминального распределения $d \mu_{\mathrm{5}}$, определенного на множестве $\mathcal{N}=\{0,1, \ldots, n\}$ равенством

$$
\mu_{\mathrm{B}}(\{x\})=\left(\begin{array}{l}
n \\
x
\end{array}\right) p^{x} q^{n-x}, \quad x \in \mathscr{N}, 0<p<1, q=1-p
$$

- такую систему в $\mathscr{L}_{2}\left(\mu_{\mathrm{B}}\right)$ образуют многочлены Кравчука

$$
K_{\alpha}(x)=\left(\begin{array}{l}
n \\
\alpha
\end{array}\right)^{-1 / 2}(p q)^{-\alpha / 2} \sum_{\nu=0}^{\alpha}(-1)^{\nu}\left(\begin{array}{l}
n-x \\
\alpha-\nu
\end{array}\right)\left(\begin{array}{l}
x \\
\nu
\end{array}\right) p^{\alpha-\nu} q^{\nu}
$$

где $\alpha=0,1, \ldots, n, x \in \mathscr{N}$.

Несложные комбинаторные преобразования позволяют получить для многочленов (1) (а также для многочленов Пуассона-Шарлье, ортогональных относительно пуассоновского распределения) новые формы записи через так называемые факториально-степенные многочлены, определение и описание свойств которых сведены в п. 1. Такие формы записи (см. п. 2) позволяют, например, технически довольно простым способом получить представления попарных произведений многочленов Кравчука или многочленов Пуассона-Шарлье в виде линейных комбинаций одноименных многочленов. С другой стороны, одна из новых форм записи многочленов Кравчука подсказывает, как может выглядеть аналог системы многочленов Кравчука для случая полиномиального распределения $d \mu_{\Pi}$, определенного на множестве

$$
\mathscr{N}^{r}=\left\{\mathbf{x}=\left(x_{0}, x_{1}, \ldots, x_{r}\right), x_{i} \in \mathcal{N}, i=0,1, \ldots, r, x_{0}+x_{1}+\cdots+x_{r}=n\right\}
$$

равенством

$$
\mu_{\Pi}(\{\mathbf{x}\})=\frac{n !}{x_{0} ! x_{1} ! \cdots x_{r} !} p_{0}^{x_{0}} p_{1}^{x_{1}} \cdots p_{r}^{x_{r}}, \quad p_{0}+p_{1}+\cdots+p_{r}=1 .
$$

В п. 3 приводится такое семейство многочленов, ортогональных относительно полиномиального распределения.

1. Факториально-степенные многочлены и факториально-степенной формализм. Сделаем несколько замечаний об обозначениях. Светлыми строчными греческими буквами будут обозначаться только целые числа. Полужирными строчными буквами будут обозначаться $r$-мерные векторы, например, $\nu$ обозначает вектор $\left(\nu_{1}, \ldots, \nu_{r}\right)$ с целочисленными координатами. Соответствугщими прописными светлыми буквами обозначаются суммы координат таких векторов, например, $\mathrm{N}=$ $\nu_{1}+\cdots+\nu_{r}$. Запись вида $\mathbf{a}^{\nu}$ обозначает произведение $a_{1}^{\nu_{1}} \cdots a_{r}^{\nu_{r}}, \mathbf{a}\langle\mathbf{a}, \mathbf{b}\rangle-$ скалярное произведение $a_{1} b_{1}+\cdots+a_{r} b_{r}$. Всюду ниже полагаем: $[x]^{\nu}=x(x-1) \cdots(x-\nu+1)$, $[x]_{\nu}=x(x+1) \cdots(x+\nu-1)$, если $\nu=1,2, \ldots,[x]^{0}=[x]_{0}=1,[x]^{\nu}=0,[x]_{\nu}=0$ при остальных значениях $\nu ; \nu !=[\nu]^{\nu}=\Gamma(\nu+1)$. Запись вида $[\mathbf{x}]^{\nu}$ обозначает произведение $\left[x_{1}\right]^{\nu_{2}} \cdots\left[x_{r}\right]^{\nu_{r}}$ и $\boldsymbol{\nu} !=[\nu]^{\nu}$. Считаем, что если у знака суммы опуцены нижний и верхнй пределы суммирования, суммирование проводится по всем целочисленным значениям индекса (индексов). 
О п р е д е л ен и е. Будем называть факториально-степеняым многочленом (ФСМ) $P_{\alpha}(\mathbf{a}, \mathbf{x} / / d ; c)$ степени $\alpha \geqslant 0$ сумму

$$
\sum_{\nu_{1}, \ldots, \nu_{r}} \frac{[\alpha]^{\nu_{1}+\cdots+\nu_{r}}}{\nu_{1} ! \cdots \nu_{r} !} a_{1}^{\nu_{1}} \cdots a_{r}^{\nu_{r}} \frac{\left[x_{1}\right]^{\nu_{1}} \cdots\left[x_{r}\right]^{\nu_{r}}}{[d]^{\nu_{1}+\cdots+\nu_{r}}} c^{\alpha-\nu_{1}-\cdots-\nu_{r}}
$$

(полагаем $d>\alpha$ и $0 /[d]^{\varkappa}=0$ при $\varkappa<0, \varkappa>d$ ) или, в краткой записи,

$$
\sum_{\nu} \frac{[\alpha]^{\mathrm{N}}}{\nu !} \mathbf{a}^{\nu} \frac{[\mathbf{x}]^{\nu}}{[d]^{\mathrm{N}}} c^{\alpha-\mathrm{N}}
$$

Для ФСМ удобна аддитивная запись в виде многочлена

$$
\left(a_{1} \frac{\left[x_{1}\right]}{[d]}+\cdots+a_{r} \frac{\left[x_{r}\right]}{[d]}+c\right)^{\alpha},
$$

поскольку общий член его разложения как обычного многочлена $\left(z_{1}+\cdots+z_{r}+c\right)^{\alpha}$ при сохранении квадратных скобок у $x_{1}, \ldots, x_{r}$ и $d\left(\right.$ так, например, $(a[x] /[d]+c)^{2}=$ $a^{2}[x]^{2} /[d]^{2}+2 a c x / d+c^{2}$, но $\left.[d]^{\nu_{i}}[d]^{\nu_{j}}=[d]^{\nu_{i}+\nu_{j}}\right)$, совпадает с общим членом суммы, фигурирующей в определении. (Отношения вида $\frac{[x]}{[d]}$ или $[x] /[d]$ иногда будем записывать для удобства в виде $\frac{x}{d}$ или $x / / d$.)

Из определения следует, что всякий ФСМ нулевой степени есть единица, а при $r=1$ ФСМ можно рассматривать как частный случай гипергеометрического ряда.

Аналогично определяется «недробная» модификация ФСМ:

$$
\left(a_{1}\left[x_{1}\right]+\cdots+a_{r}\left[x_{r}\right]+c\right)^{\alpha}=\sum_{\nu} \frac{[\alpha]^{\mathrm{N}}}{\nu !} \mathbf{a}^{\nu}[\mathbf{x}]^{\nu} c^{\alpha-\mathrm{N}}
$$

Особенности предлагаемого факториально-степенного формализма иллюстрируют очевидные соотношения типа $e^{[\alpha]}=2^{\alpha}$ (и, вообще, $\left.b^{\alpha} \exp \left\{\frac{a}{b}[\alpha]\right\}=(a+b)^{\alpha}\right)$,

и

$$
\begin{gathered}
b^{\alpha}(a[\alpha]+b)^{\beta}=b^{\beta}(a[\beta]+b)^{\alpha} \text { и } b^{\alpha}\left(a \frac{\alpha}{\beta}+b\right)^{\gamma}=b^{\gamma}\left(a \frac{\gamma}{\beta}+b\right)^{\alpha} \\
(a[x]+b[y]+c)^{\alpha}=a^{\alpha}\left([x]+\frac{b}{a}[y]+\frac{c}{a}\right)^{\alpha}
\end{gathered}
$$

$$
\left(a \frac{x}{d}+b \frac{y}{d}+c\right)^{\alpha}=a^{\alpha}\left(\frac{x}{d}+\frac{b}{a} \frac{y}{d}+\frac{c}{a}\right)^{\alpha} \text {. }
$$

Отметим два важных свойства ФСМ.

Лемма 1. Справедливо тождество

$$
P_{\alpha}\left(\mathbf{a}, \mathbf{x} / / d ; c_{1}+c_{2}\right)=\sum_{\nu} \frac{[\alpha]^{\nu}}{\nu !} P_{\nu}\left(\mathbf{a}, \mathbf{x} / / d ; c_{1}\right) c_{2}^{\alpha-\nu} .
$$

Это тождество есть следствие, например, того факта, что производящая функция $\sum_{\alpha} P_{\alpha} t^{\alpha} / \alpha$ ! имеет вид $e^{c t} A(t)$, где $A(t)=\sum_{\nu}[\mathbf{x}]^{\nu}(t \mathbf{a})^{\nu} /\left(\nu ![d]^{N}\right)$, и значит, ФСМ как многочлены по $c$ суть многочлены Аппеля. Для них это свойство хорошо известно. Отметим, впрочем, что это свойство можно рассматривать и как тривиальный частный случай наиболее общего на сегодня результата такого рода - биномиальной теоремы для многочленов Шеффера (включаюших в себя многочлены Аппеля), см. $[10$, с. 700 и с. 736$]$.

Лемма 2. Справедливо тождество

$$
(a[x+y]+b[z])^{\alpha}=(a[x]+a[y]+b[z])^{\alpha} .
$$

Действительно, пользуясь формулой свертки Вандермонда, имеем:

$$
\begin{aligned}
(a[x+y]+b[z])^{\alpha} & =\sum_{\nu=0}^{\alpha} \frac{[\alpha]^{\nu}}{\nu !} a^{\nu}[x+y]^{\nu} b^{\alpha-\nu}[z]^{\alpha-\nu} \\
& =\sum_{\nu=0}^{\alpha} \frac{[\alpha]^{\nu}}{\nu !} a^{\nu} b^{\alpha-\nu}[z]^{\alpha-\nu} \sum_{\mu=0}^{\nu} \frac{[\nu]^{\mu}}{\mu !}[x]^{\mu}[y]^{\nu-\mu}
\end{aligned}
$$




$$
\begin{aligned}
& =\sum_{\mu=0}^{\alpha} \sum_{\nu=0}^{\alpha-\mu} \frac{[\alpha]^{\nu+\mu}}{\nu ! \mu !} a^{\nu} a^{\mu} b^{\alpha-\nu-\mu}[x]^{\mu}[y]^{\nu}[z]^{\alpha-\nu-\mu} \\
& =\sum_{\mu=0}^{\alpha} \frac{[\alpha]^{\mu}}{\mu !} a^{\mu}[x]^{\mu} \sum_{\nu=0}^{\alpha-\mu} \frac{[\alpha-\mu]^{\nu}}{\nu !} a^{\nu}[y]^{\nu} b^{\alpha-\nu-\mu}[z]^{\alpha-\nu-\mu} \\
& =\sum_{\mu=0}^{\alpha} \frac{[\alpha]^{\mu}}{\mu !} a^{\mu}[x]^{\mu}(a[y]+b[z])^{\alpha-\mu}=(a[x]+a[y]+b[z])^{\alpha} .
\end{aligned}
$$

Утверждение этой леммы очевидным образом распространяется на ФСМ общего вида.

Приведем еще одно комбинаторное тождество, касающееся ФСМ.

Лемма 3 (тождество М. Лерха). Справедливо пождество

$$
\frac{[x]^{\alpha}}{[d]^{\alpha}}=\sum_{\nu} \frac{[\alpha]^{\nu}[d-x]^{\nu}}{\nu ![d]^{\nu}}(-1)^{\nu}
$$

Это тождество было приведено и доказано впервые, по-видимому, в [9] (см. также $[5$, с. 61 , тождество 11]). Несколько обобщенный вариант этого тождества легко доказывается при помощи формулы свертки Вандермонда для возрастающих факториальных степеней $(\beta \geqslant \alpha)$ :

$$
\begin{aligned}
\frac{[a]^{\alpha}}{[b]^{\beta}} & =\frac{[a-\alpha+1]_{\alpha}}{[b-\beta+1]_{\beta}}=\frac{[b-\beta+1-(b-a-\beta+\alpha)]_{\alpha}}{[b-\beta+1]_{\beta}} \\
& =\sum_{\nu} \frac{[\alpha]^{\nu}}{\nu !}(-1)^{\nu}[b-a-\beta+\alpha]^{\nu} \frac{[b-\beta+1]_{\alpha-\nu}}{[b-\beta+1]_{\beta}} \\
& =\sum_{\nu} \frac{[\alpha]^{\nu}}{\nu !} \frac{[b-a-(\beta-\alpha)]^{\nu}}{[b]^{\nu+(\beta-\alpha)}}(-1)^{\nu} .
\end{aligned}
$$

Не лишен интереса тот факт, что тождество Лерха дает обоснование группе из трех формальных преобразований, выделенных фигурными скобками в цепочке преобразований

$$
\frac{[x]^{\alpha}}{[d]^{\alpha}}=\left\{\left(\frac{x}{d}\right)^{\alpha}=\left(\frac{-(d-x)+d}{d}\right)^{\alpha}=\left(-\frac{d-x}{d}+1\right)^{\alpha}\right\}=\sum_{\nu} \frac{[\alpha]^{\nu}[d-x]^{\nu}}{\nu ![d]^{\nu}}(-1)^{\nu} \text {. }
$$

2. Многочлены Кравчука как ФСМ. Разлагая в правой части (1) сомножители $q^{\nu}=(1-p)^{\nu}$ по формуле бинома Ньютона, меняя порядок суммирования и пользуясь формулой свертки Вандермонда (см. замечание 3 ниже), перепишем многочлены (1) в виде

$$
K_{\alpha}(x)=k_{\alpha}\left(\frac{x}{\bar{n}}-p\right)^{\alpha}, \quad \text { где } \quad k_{\alpha}=(-1)^{\alpha}\left(\frac{[n]^{\alpha}}{\alpha ! p^{\alpha} q^{\alpha}}\right)^{1 / 2} .
$$

Таким образом, $K_{\alpha}(x)=k_{\alpha} P_{\alpha}(1, x / / n ;-p)$. По существу, та же форма записи многочленов $K_{\alpha}(x)$ (правда, без нормировки) приведена в [7, формула (2.20)], где многочлены Кравчука трактуются как гипергеометрические функции ${ }_{2} P_{1}\left(-\alpha,-x ;-n ; p^{-1}\right)$.

Форма записи (4) удобна, в частности, тем, что при помощи лемм 1 и 3 она позволяет технически довольно просто получать линейные разложения произведений $K_{\alpha}(x) K_{\beta}(x)$ многочленов Кравчука по одноименной системе.

Теорема 1. Имеет место разложение

$$
K_{\alpha}(x) K_{\beta}(x)=\left(\frac{p^{\alpha+\beta}[n]^{\alpha}[n]^{\beta}}{q^{\alpha+\beta} \alpha ! \beta !}\right)^{1 / 2} \sum_{\gamma} l_{\gamma} K_{\gamma}(x),
$$

где коэффициенты $l_{\gamma}$ имегт вид

$$
\frac{(-1)^{\gamma} q^{\gamma / 2}}{\sqrt{\gamma ! p^{\gamma}[n]^{\gamma}}} \sum_{\theta, \delta} \frac{(-1)^{\delta}}{\theta ! \delta ! p^{\theta}[n]^{\theta+\delta}} \sum_{\nu, \mu} \frac{[\alpha]^{\nu}[\beta]^{\mu}[\nu+\mu-\theta]^{\gamma}}{(\nu-\theta-\delta) !(\mu-\theta-\delta) !}(-1)^{\nu+\mu}
$$


Д о к а з а т е л в с т в о. По формуле (6) и определению ФСМ

$$
\begin{aligned}
K_{\alpha}(x) K_{\beta}(x) & =k_{\alpha} k_{\beta}\left(\frac{x}{\bar{n}}-p\right)^{\alpha}\left(\frac{x}{\bar{n}}-p\right)^{\beta} \\
& =k_{\alpha} k_{\beta} \sum_{\nu, \mu} \frac{[\alpha]^{\nu}[\beta]^{\mu}}{\nu ! \mu !} \frac{[x]^{\nu}[x]^{\mu}}{[n]^{\nu}[n]^{\mu}}(-p)^{\alpha+\beta-\nu-\mu} .
\end{aligned}
$$

При помощи формулы свертки Вандермонда получаем:

$$
\begin{aligned}
{[x]^{\nu}[x]^{\mu} } & =[x]^{\nu}[\nu+x-\nu]^{\mu}=[x]^{\nu} \sum_{\theta}[\mu]^{\theta}[\nu]^{\theta}[x-\nu]^{\mu-\theta} / \theta ! \\
& =\sum_{\theta}[\mu]^{\theta}[\nu]^{\theta}[x]^{\nu+\mu-\theta} / \theta !
\end{aligned}
$$

Поэтому

$$
\frac{[x]^{\nu}[x]^{\mu}}{[n]^{\nu}[n]^{\mu}}=\sum_{\theta} \frac{[\mu]^{\theta}[\nu]^{\theta}}{\theta !} \frac{[n]^{\nu+\mu-\theta}}{[n]^{\nu}[n]^{\mu}} \frac{[x]^{\nu+\mu-\theta}}{[n]^{\nu+\mu-\theta}} .
$$

Пользуясь леммой 1 , преобразуем последнюю дробь в правой части (7):

$$
\begin{aligned}
\frac{[x]^{\nu+\mu-\theta}}{[n]^{\nu+\mu-\theta}} & =\left(\frac{x}{n}\right)^{\nu+\mu-\theta}=\left(\frac{x}{\bar{n}}-p+p\right)^{\nu+\mu-\theta} \\
& =\sum_{\gamma} \frac{[\nu+\mu-\theta]^{\gamma}}{\gamma !}\left(\frac{x}{\bar{n}}-p\right)^{\gamma} p^{\nu+\mu-\theta-\gamma} \\
& =\sum_{\gamma}(-1)^{\gamma}[\nu+\mu-\theta]^{\gamma} p^{\nu+\mu-\theta}\left(\frac{q^{\gamma}}{\gamma ! p^{\gamma}[n]^{\gamma}}\right)^{1 / 2} K_{\gamma}(x) .
\end{aligned}
$$

Теперь для того чтобы прийти к разложению (5), остается, пользуясь правилом (3), преобразовать предпоследнюю дробь в правой части (7):

$$
\begin{aligned}
\frac{[n]^{\nu+\mu-\theta}}{[n]^{\nu}[n]^{\mu}} & =\frac{[n]^{\nu}[n-\nu]^{\mu-\theta}}{[n]^{\nu}[n]^{\theta}[n-\theta]^{\mu-\theta}}=\frac{1}{[n]^{\theta}}\left(\frac{n-\nu}{n-\theta}\right)^{\mu-\theta} \\
& =\frac{1}{[n]^{\theta}}\left(\frac{-(\nu-\theta)+n-\theta}{n-\theta}\right)^{\mu-\theta}=\frac{1}{[n]^{\theta}}\left(-\frac{\nu-\theta}{\overline{n-\theta}}+1\right)^{\mu-\theta} \\
& =\frac{1}{[n]^{\theta}} \sum_{\delta} \frac{[\mu-\theta]^{\delta}[\nu-\theta]^{\delta}}{\delta ![n-\theta]^{\delta}}(-1)^{\delta}=\sum_{\delta} \frac{[\nu-\theta]^{\delta}[\mu-\theta]^{\delta}}{\delta ![n]^{\theta+\delta}}(-1)^{\delta}
\end{aligned}
$$

и изменить порядок суммирования.

Теорема 1 доказана.

3 а м е ч а н и е 1. Пользуясь записью (4) многочленов $K_{\alpha}(x)$ в виде ФСМ, при

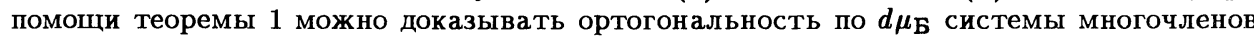
Кравчука, не прибегая к производящей функции, как это обычно делается. Действительно (ниже $\beta_{n, p}-$ случайная величина с распределением $d \mu_{\mathrm{5}}$ ),

$$
\mathbf{M}\left(\frac{\beta_{n, p}}{n}-p\right)^{\alpha}=\sum_{\nu} \frac{[\alpha]^{\nu}}{\nu !} \frac{\mathbf{M}\left[\beta_{n, p}\right]^{\nu}}{[n]^{\nu}}(-p)^{\alpha-\nu}=0^{\alpha}=\delta_{0 \alpha}
$$

поскольку $\mathbf{M}\left[\beta_{n, p}\right]^{\nu}=p^{\nu}[n]^{\nu}$. Поэтому в силу (5) имеем:

где

$$
\mathbf{M} K_{\alpha}\left(\beta_{n, p}\right) K_{\beta}\left(\beta_{n, p}\right)=(-1)^{\alpha+\beta}\left(\frac{p^{\alpha+\beta}[n]^{\alpha}[n]^{\beta}}{q^{\alpha+\beta} \alpha ! \beta !}\right)^{1 / 2} l_{0},
$$

$$
l_{0}=\sum_{\theta, \delta} \frac{(-1)^{\delta}}{\theta ! \delta ! p^{\theta}[n]^{\theta+\delta}} \sum_{\nu, \mu} \frac{[\alpha]^{\nu}[\beta]^{\mu}}{(\nu-\theta-\delta) !(\mu-\theta-\delta) !}(-1)^{\nu+\mu}
$$

и, так как

$$
\begin{aligned}
\sum_{\nu} \frac{[\alpha]^{\nu}}{(\nu-\theta-\delta) !}(-1)^{\nu} & =[\alpha]^{\theta+\delta}(-1)^{\theta+\delta} \sum_{\nu} \frac{[\alpha-\theta-\delta]^{\nu-\theta-\delta}}{(\nu-\theta-\delta) !}(-1)^{\nu-\theta-\delta} \\
& =[\alpha]^{\theta+\delta}(-1)^{\theta+\delta} 0^{\alpha-(\theta+\delta)}
\end{aligned}
$$


а сумма по $\mu$ сворачивается так же, то $l_{0}=\delta_{\alpha \beta} q^{\alpha} \alpha ! /\left(p^{\alpha}[n]^{\alpha}\right)$ и, значит, $\mathrm{M} K_{\alpha}\left(\beta_{n, p}\right) K_{\beta}\left(\beta_{n, p}\right)=\delta_{\alpha \beta}$.

3 а м е ч а н и е 2. Многочлены $P C_{\alpha}(x)$ Пуассона-Шарлье (см., например, [3, $\S 2.81])$, ортогональные относительно пуссоновского распределения $d \mu_{P}\left(\mu_{P}(\{x\})=\right.$ $\left.\lambda^{x} e^{-\lambda} / x !, x=0,1, \ldots\right)$, как легко проверить, можно записать при помощи «недробных» ФСМ следующим образом:

$$
P C_{\alpha}(x)=\sqrt{\frac{\lambda^{\alpha}}{\alpha !}}\left(\frac{1}{\lambda}[x]-1\right)^{\alpha}=\frac{1}{\sqrt{\alpha ! \lambda^{\alpha}}}([x]-\lambda)^{\alpha} .
$$

Поскольку утверждение леммы 1 , очевидно, остается справедливым и для «недробных» ФСМ, можно, повторяя выкладки доказательства теоремы 1 , получить аналогичное (5) линейное разложение произведения двух многочленов Пуассона-Шарлье:

где

$$
P C_{\alpha}(x) P C_{\beta}(x)=\left(\frac{\lambda^{\alpha+\beta}}{\alpha ! \beta !}\right)^{1 / 2} \sum_{\gamma} \tilde{l}_{\gamma} P C_{\gamma}(x),
$$

$$
\tilde{l}_{\gamma}=\frac{1}{\sqrt{\gamma^{!} \lambda^{\gamma}}} \sum_{\theta} \frac{1}{\theta ! \lambda^{\theta}} \sum_{\nu, \mu} \frac{[\alpha]^{\nu}[\beta]^{\mu}[\nu+\mu-\theta]^{\gamma}}{(\nu-\theta) !(\mu-\theta) !}(-1)^{\nu+\mu} .
$$

Это разложение, как и разложение (5), дает возможность доказывать ортогональ-

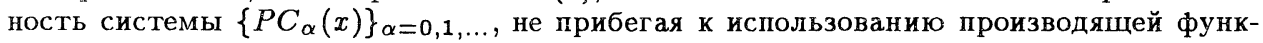
ции.

3 а м е ч н и е 3. Многочлены Кравчука допускают и «недробные» ФСМпредставления, например, следующего вида:

$$
K_{\alpha}(x)=(-1)^{\alpha} \tilde{k}_{\alpha}(q[x]-p[n-x])^{\alpha}=\tilde{k}_{\alpha}(p[n-x]-q[x])^{\alpha},
$$

где $\tilde{k}_{\alpha}=\left([n]^{\alpha} \alpha ! p^{\alpha} q^{\alpha}\right)^{-1 / 2}$. В самом деле,

$$
\begin{aligned}
\left(\frac{x}{n}-p\right)^{\alpha} & =\sum_{\nu=0}^{\alpha} \frac{[\alpha]^{\nu}}{\nu !} \frac{[x]^{\nu}}{[n]^{\nu}}(-p)^{\alpha-\nu}=\frac{1}{[n]^{\alpha}} \sum_{\nu=0}^{\alpha} \frac{[\alpha]^{\nu}}{\nu !}[x]^{\nu}[n-\nu]^{\alpha-\nu}(-p)^{\alpha-\nu} \\
& =\frac{1}{[n]^{\alpha}} \sum_{\nu=0}^{\alpha} \frac{[\alpha]^{\nu}}{\nu !}[x]^{\nu}[n-x+x-\nu]^{\alpha-\nu}(-p)^{\alpha-\nu} \\
& =\frac{1}{[n]^{\alpha}} \sum_{\nu=0}^{\alpha} \sum_{\mu=0}^{\alpha-\nu} \frac{[\alpha]^{\nu}}{\nu !}[x]^{\nu} \frac{[\alpha-\nu]^{\mu}}{\mu !}[n-x]^{\mu}[x-\nu]^{\alpha-\nu-\mu}(-p)^{\alpha-\nu} \\
& =\frac{1}{[n]^{\alpha}} \sum_{\mu=0}^{\alpha} \frac{[\alpha]^{\mu}}{\mu !}[n-x]^{\mu}[x]^{\alpha-\mu}(-p)^{\alpha} \sum_{\nu=0}^{\alpha-\mu} \frac{[\alpha-\mu]^{\nu}}{\nu !}(-p)^{-\nu} \\
& =\frac{(-p)^{\alpha}}{[n]^{\alpha}} \sum_{\mu=0}^{\alpha} \frac{[\alpha]^{\mu}}{\mu !}[n-x]^{\mu}(-q)^{\alpha-\mu}[x]^{\alpha-\mu} p^{\mu-\alpha} \\
& =\frac{(-1)^{\alpha}}{[n]^{\alpha}}(p[n-x]-q[x])^{\alpha}=\frac{1}{[n]^{\alpha}}\left(q[x]^{\alpha}-p[n-x]\right)^{\alpha} .
\end{aligned}
$$

3. Факториально-степенные многочлены, ортогональные относительно полиномиального распределения.

Tеорема 2. $\Phi C M$ еида

$$
h_{\alpha}\left\langle\mathbf{a}, \frac{\mathbf{x}}{\bar{n}}-\mathbf{p}\right\rangle^{\alpha}=h_{\alpha}\left(a_{1} \frac{x_{1}}{n}+\cdots+a_{r} \frac{x_{r}}{n}-c\right)^{\alpha},
$$

где $h_{\alpha}=\left([n]^{\alpha} /\left(\alpha !\left(a_{1}^{2} p_{1}+\cdots+a_{r}^{2} p_{r}-c^{2}\right)^{\alpha}\right)^{1 / 2}\right) u c=a_{1} p_{1}+\cdots+a_{r} p_{r}, a$ параметры $a_{1}, \ldots, a_{r}-$ произвольнье вещественные иисла, образуют семейство $\left\{P_{\alpha}(x)\right\}_{\alpha=0,1, \ldots, n}$ многочленов, ортогональньх относительно полияомиального распределения (2). 
Д о к а з а т е л ь с т в о. Из определения ФСМ имеем (ниже $\pi_{n, p}$ - случайная векторнозначная величина, имеюшая полиномиальное распределение (2)):

$$
\frac{\mathbf{M} P_{\alpha}\left(\pi_{n, \mathbf{p}}\right) P_{\beta}\left(\boldsymbol{\pi}_{n, \mathbf{p}}\right)}{h_{\alpha} h_{\beta}}=\sum_{\nu, \mu} \frac{[\alpha]^{\mathrm{N}}[\beta]^{\mathbf{M}}}{\nu ! \mu !} \mathbf{a}^{\nu+\mu}(-c)^{\alpha+\beta-\mathrm{N}-\mathbf{M}} \frac{\mathbf{M}\left[\boldsymbol{\pi}_{n, \mathbf{p}}\right]^{\nu}\left[\boldsymbol{\pi}_{n, \mathbf{p}}\right]^{\mu}}{[n]^{\mathrm{N}}[n]^{\mathbf{M}}}
$$

Пользуясь преобразованиями (6), (8) и тем фактом, что $\mathbf{M}\left[\pi_{1}\right]^{\nu_{1}} \ldots\left[\pi_{r}\right]^{\nu_{r}}=$ $p_{1}^{\nu_{1}} \cdots p_{r}^{\nu_{r}}[n]^{\nu_{1}}+\cdots+\nu_{r}$, перепишем это выражение следующим образом:

$$
\begin{aligned}
& \sum_{\nu, \mu, \theta} \frac{[\alpha]^{\mathrm{N}}[\beta]^{\mathrm{M}}}{\theta !(\boldsymbol{\nu}-\boldsymbol{\theta}) !(\mu-\theta) !} \mathbf{a}^{\nu+\mu}(-c)^{\alpha+\beta-\mathrm{N}-\mathrm{M}} \frac{\mathrm{M}\left[\boldsymbol{\pi}_{n, \mathbf{p}}\right]^{\nu+\mu-\theta}}{[n]^{\mathrm{N}}[n]^{\mathrm{M}}} \\
& =\sum_{\nu, \mu, \theta} \frac{[\alpha]^{\mathrm{N}}[\beta]^{\mathrm{M}}}{\boldsymbol{\theta} !(\nu-\theta) !(\mu-\theta) !} \mathbf{a}^{\nu+\mu} \mathbf{p}^{\nu+\mu-\theta}(-c)^{\alpha+\beta-\mathrm{N}-\mathrm{M}} \frac{[n]^{\mathrm{N}+\mathrm{M}-\Theta}}{[n]^{\mathrm{N}}[n]^{\mathrm{M}}} \\
& =\sum_{\nu, \mu, \theta, \gamma} \frac{[\alpha]^{\mathrm{N}}[\beta]^{\mathrm{M}}[\mathrm{N}-\Theta]^{\gamma}[\mathrm{M}-\Theta]^{\gamma}(-1)^{\gamma}}{\theta !(\nu-\theta) !(\mu-\theta) ! \gamma ![n]^{\Theta+\gamma}} \mathbf{a}^{\nu+\mu} \mathbf{p}^{\nu+\mu-\theta}(-c)^{\alpha+\beta-\mathrm{N}-\mathrm{M}} .
\end{aligned}
$$

Последкее выражєние перепишем в виде

где

$$
\sum_{\theta, \gamma} \frac{(-1)^{\gamma} \mathbf{p}^{-\theta}}{\theta ! \gamma ![n]^{\Theta+\gamma}} A(\alpha, \theta, \gamma) A(\beta, \boldsymbol{\theta}, \gamma)
$$

$$
\begin{aligned}
& A(\alpha, \theta, \gamma)=\sum_{\nu} \frac{[\alpha]^{\mathrm{N}}[\mathrm{N}-\Theta]^{\gamma}}{(\nu-\theta) !} \mathbf{a}^{\nu} \mathbf{p}^{\nu}(-c)^{\alpha-\mathrm{N}} \\
& =\sum_{\nu, \delta: \Delta=\gamma} \frac{[\alpha]^{\mathrm{N}}[\nu-\boldsymbol{\theta}]^{\delta} \gamma !}{\delta !(\nu-\theta) !} \mathbf{a}^{\nu} \mathbf{p}^{\nu}(-c)^{\alpha-\mathrm{N}}=\sum_{\nu, \delta: \Delta=\gamma} \frac{[\alpha]^{\mathrm{N}} \gamma !}{\delta !(\nu-\boldsymbol{\theta}-\boldsymbol{\delta}) !} \mathbf{a}^{\nu} \mathbf{p}^{\nu}(-c)^{\alpha-\mathrm{N}} \\
& =\sum_{\delta: \Delta=\gamma} \frac{\gamma !}{\delta !}[\alpha]^{\Theta+\Delta} \mathbf{a}^{\theta+\delta} \mathbf{p}^{\theta+\delta} \sum_{\nu} \frac{[\alpha-\Theta-\Delta]^{\mathrm{N}-\Theta-\Delta}}{(\nu-\theta-\delta) !} \mathbf{a}^{\nu-\theta-\delta} \mathbf{p}^{\nu-\theta-\delta}(-c)^{\alpha-\mathrm{N}} \\
& =\sum_{\delta: \Delta=\gamma} \frac{\gamma !}{\delta !}[\alpha]^{\Theta+\Delta_{\mathbf{a}}}{ }^{\boldsymbol{\theta}+\boldsymbol{\delta}_{\mathbf{p}}} \mathbf{p}^{\boldsymbol{\theta}+\boldsymbol{\delta}_{0}} 0^{\alpha-\odot-\Delta} \\
& =[\alpha]^{\Theta+\gamma} 0^{\alpha-\gamma-\Theta} \mathbf{a}^{\theta} \mathbf{p}^{\theta} \sum_{\delta_{1}+\cdots+\delta_{r}=\gamma} \frac{\gamma !}{\delta_{1} ! \cdots \delta_{r} !}\left(a_{1} p_{1}\right)^{\delta_{1}} \cdots\left(a_{r} p_{r}\right)^{\delta_{r}} \\
& =[\alpha]^{\Theta+\gamma} 0^{\alpha-\gamma-\Theta} \mathbf{a}^{\theta} \mathbf{p}^{\theta} c^{\gamma} .
\end{aligned}
$$

Из полученного видио, что $A(\alpha, \boldsymbol{\theta}, \gamma)=0$ при $\Theta=\theta_{1}+\cdots+\theta_{r} \neq \alpha-\gamma, A(\beta, \boldsymbol{\theta}, \gamma)=0$ при $\Theta \neq \beta-\gamma, A(\alpha, \theta, \gamma) A(\beta, \theta, \gamma)=0$ при $\alpha \neq \beta$. Следовательно,

$$
\begin{aligned}
\frac{\mathbf{M} P_{\alpha}\left(\boldsymbol{\pi}_{n, \mathbf{p}}\right) P_{\beta}\left(\boldsymbol{\pi}_{n, \mathbf{p}}\right)}{h_{\alpha} h_{\beta}} & =\sum_{\boldsymbol{\theta}, \gamma} \frac{(-1)^{\gamma} \mathbf{p}^{-\boldsymbol{\theta}}}{\boldsymbol{\theta} \boldsymbol{\gamma} ![n]^{\Theta+\gamma}}\left(\mathbf{a}^{\theta} \mathbf{p}^{\boldsymbol{\theta}} c^{\gamma}\right)^{2}[\alpha]^{\Theta+\gamma}[\beta]^{\Theta+\gamma} 0^{\alpha-\gamma-\Theta} 0^{\beta-\gamma-\Theta} \\
& =\delta_{\alpha \beta} \sum_{\gamma, \boldsymbol{\theta}} \frac{(-1)^{\gamma}}{\boldsymbol{\theta} ! \gamma ![n]^{\alpha}} \mathbf{a}^{2 \boldsymbol{\theta}} \mathbf{p}^{\boldsymbol{\theta}} c^{2 \gamma}(\alpha !)^{2} \\
& =\delta_{\alpha \beta} \frac{\alpha !}{[n]^{\alpha}} \sum_{\gamma} \frac{\alpha !}{\gamma !(\alpha-\gamma) !}\left(-c^{2}\right)^{\gamma} \sum_{\boldsymbol{\theta}: \Theta=\alpha-\gamma} \frac{(\alpha-\gamma) !}{\boldsymbol{\theta} !}\left(\mathbf{a}^{2} \mathbf{p}\right)^{\theta} \\
& =\delta_{\alpha \beta} \frac{\alpha !}{[n]^{\alpha}} \sum_{\gamma} \frac{[\alpha]^{\gamma}}{\gamma !}\left(-c^{2}\right)^{\gamma}\left(a_{1}^{2} p_{1}+\cdots+a_{r}^{2} p_{r}\right)^{\alpha-\gamma} \\
& =\delta_{\alpha \beta} \frac{\alpha !}{[n]^{\alpha}}\left(a_{1}^{2} p_{1}+\cdots+a_{r}^{2} p_{r}-c^{2}\right)^{\alpha}=\delta_{\alpha \beta} \frac{1}{h_{\alpha}^{2}} .
\end{aligned}
$$

Теорема 2 доказана. 


\section{СПИСОК ЛИТЕРАТУРЫ}

1. Висков О.В., Прохоров Ю.В., Хохлов В.И. Пуассоновский аналог неравенства Чернова. - Обозрение прикл. и промышл. матем., 2001, т. 8, в. 1, с. 128-129.

2. Прохоров Ю. В., Висков О.В., Хохлов В. И. Биномиальные аналоги неравенства Чернова. - Теория вероятн. и ее примен., 2001, т. 46, в. 3, с. 592-594.

3. Сеге Г. Ортогональные многочлены. М.: Физматгиз, 1962, 500 с.

4. Chernoff $H$. A note on an inequality involving the normal distribution. - Ann. Probab., 1981, v. 9, p. 533-535.

5. Kaucký J. Kombinatorické identity. Bratislava: J. Kaucký/Veda, 1975, 476 s.

6. Khokhlov V.I. Factorial-power polynomials that are orthogonal with respect to the multinomial distribution. - В сб: Пятая международная Вильнюсская конференция по теории вероятностей и математической статистике. Тезисы докладов. Т. 1. Вильнюс: ИМК АН ЛитССР, 1989, с. 254-255.

7. Koornwinder T.H. Krawtchouk polynomials, a unification of two different group theoretic interpretations. - SIAM J. Math. Anal., 1982, v. 13, № 6, p. 1011-1023.

8. Krawtchouk M. Sur une généralisation des polynomes d'Hermite. - C. r. hebd. séances l'Acad. Sci., 1929, t. 189, № 17, p. 620-622.

9. Lerch M. Z počtu integrálního. - Rozpravy Čes. Akad. cís. Fr. Jos. pro vědy, slovesnost a umení v Praze. Trída II. Řada mat.-fys., 1893, sv. 2, s. 9, s. 1-40.

10. Rota G.-C., Kahaner D., Odlyzko A. On the foundations of combinatorial theory. VIII. Finite operator calculus. - J. Math. Anal. Appl., 1973, v. 42, № 3, p. 684-760.

Поступила в редакцию 18.I.1985

Переработанный вариант 1.VIII. 2001

(C) 2001 г. ПРОХОРОВ Ю. В. БИНОМИАЛЬНЫЕ АНАЛОГИ НЕРАВЕНСТВА ЧЕРНОВА

Ключевые слова и фразы: биномиальное распределение, моментные неравенства, многочлены Кравчука, факториально-степенной формализм, комбинаторные тождества.

В [1] была установлена справедливость следующего неравенства, являющегося пуассоновским аналогом неравенства [3] Чернова. Если $\pi_{\lambda}-$ случайная величина, имеющая пуассоновское распределение с параметром $\lambda$, то для произвольной функции $g(\cdot)$ выполняется неравенство $\lambda \mathbf{M}\left(g\left(\pi_{\lambda}+1\right)-g\left(\pi_{\lambda}\right)\right)^{2} \geqslant \mathbf{D} g\left(\pi_{\lambda}\right)$, причем равенство достигается тогда и только тогда, когда $g(\cdot)$ - линейная функция своего аргумента.

Покажем, что имеют место следующие биномиальные аналоги этих неравенств.

Теорема 1. Пусть $\beta_{n, p}$ - случайная величина, имеющая биномиальное распределение с параметрами $n$ и $p$. Для произвольного многочлена $g(\cdot)$ степени не выие $n$ выполняются неравенства

$$
\begin{aligned}
n p q \mathbf{M}\left(g\left(\beta_{n, p}+1\right)-g\left(\beta_{n, p}\right)\right)^{2} & \geqslant \mathbf{D} g\left(\beta_{n, p}\right), \\
n p q \mathbf{M}\left(V\left(g\left(\beta_{n, p}\right)\right)\right)^{2} & \geqslant \mathbf{D} g\left(\beta_{n, p}\right),
\end{aligned}
$$

где $V(g(x))=q^{-1} \sum_{\theta=0}^{\infty}(-p / q)^{\theta}(g(x+\theta+1)-g(x+\theta)), p<q, p+q=1$, причем равенства в (1) и (2) достигаются тогда и только тогда, когда $g(\cdot)$ - линейная функии своего аргумента.

* Математический институт им. В. А. Стеклова РАН, ул. Губкина, 8, 117966 Москва, ГСП-1, Россия; e-mail: tvp@tvp.ru 